IASSNS-HEP-93/21

IASSNS-AST 93/20

May 1993

\title{
THE RATE OF THE PROTON-PROTON REACTION
}

\author{
MARC KAMIONKOWSKI $^{\star \dagger}$ AND JOHN N. BAHCALL ${ }^{\ddagger}$ \\ School of Natural Sciences, Institute for Advanced Study, Princeton, NJ 08540
}

\begin{abstract}
We re-evaluate the nuclear matrix element for the proton-proton reaction $p+p \rightarrow{ }^{2} \mathrm{H}+e^{+}+\nu_{e}$, which is important for stellar-evolution calculations referring to stars with masses $\lesssim 1 M_{\odot}$ and for the solar-neutrino problem. We selfconsistently determine the effect of vacuum polarization on the matrix element by first correcting the low-energy scattering data to account for vacuum polarization. We then calculate the proton-proton wave function by integrating the Schrodinger equation with vacuum polarization included. We use improved data for proton-proton scattering and for the deuteron wave function. We evaluate the uncertainties that are due to experimental error and estimate those that are due to theoretical inadequacies. Without vacuum polarization, we find that the square of the overlap integral is $\Lambda^{2}=6.96$ with an uncertainty of $0.2 \%$ due to errors in the experimental parameters and an uncertainty of $1 \%$ due to lack of knowledge of the shape of the nuclear potentials. We estimate the theoretical uncertainty by using six different deuteron potentials and five different proton-proton potentials. Vacuum polarization decreases the calculated value by $0.6_{-0.4}^{+0.1 \%}$. The complete result is $\Lambda^{2}=6.92 \times\left(1 \pm 0.002_{-0.009}^{+0.014}\right)$ where the first uncertainty is due to experimental errors and the second uncertainty is due to theoretical uncertainties. Our value of $\Lambda^{2}$ is $2 \%$ smaller than the value obtained in 1969 by Bahcall and May. The improved calculations of the rate of the $p p$ reaction described here increase slightly the predicted event rates for the chlorine and the Kamiokande solar-neutrino experiments.
\end{abstract}

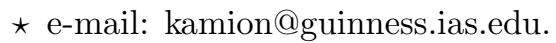

$\dagger$ SSC Fellow

$\ddagger$ e-mail: bahcall@guinness.ias.edu
} 


\section{INTRODUCTION}

The cross section for the basic reaction that initiates the proton-proton fusion chain, $p+p \rightarrow{ }^{2} \mathrm{H}+e^{+}+\nu_{e}$, is important for calculations of the stellar evolution of main-sequence stars of solar mass or less. In particular, the calculated flux of the crucial ${ }^{8} \mathrm{~B}$ solar neutrinos depends approximately upon the -2.6th power of the square of the $p p$ matrix element (Bahcall and Ulrich 1988). The rate for the $p p$ reaction was first estimated by Bethe and Critchfield (1938), and then calculated with an effective-range approximation by Salpeter (1952). The results of detailed numerical calculations were presented by Bahcall and May (1969); various specific calculations and corrections have been published since then (Brolley 1971; Gari and Huffman 1972; Gari 1978; Bargholtz 1979; Guessoum 1988; Gould and Guessoum 1990; and Carlson et al. 1991).

In this paper we re-calculate the nuclear matrix element for the $p p$ reaction using improved data and an explicit and consistent treatment of vacuum-polarization effects. We also identify and evaluate the various sources of uncertainty.

The low-energy cross-section factor for the $p p$ reaction can be written (see Bahcall et al., 1982)

$$
S_{p p}(0)=3.89\left(\frac{\Lambda^{2}}{6.92}\right)\left(\frac{G_{A} / G_{V}}{1.2573}\right)^{2}\left(\frac{1+\delta}{1.01}\right)^{2} \times 10^{-25} \mathrm{MeV}-\text { barns. }
$$

The value of $G_{A} / G_{V}=1.2573(28)$ (Hikasa et al. 1992) used here represents a weighted average of five precise modern experiments in which the correlation between the electron momentum and the neutron spin or the proton recoil spectrum are measured. The quantity $\delta=0.01_{-0.006}^{+0.02}$ (Bahcall and Pinsonneault 1992) is the fractional correction to the nuclear matrix element due to the exchange of $\pi$ and $\rho$ mesons. We do not re-evaluate $\delta$ in this paper (for a discussion of the mesonic corrections, see, e.g., Blin-Stoyle and Papageorgiou 1965, Gari and Huffman 1972, Dautry, Rho, and Riska 1976, Bargholtz 1979, and Carlson et al. 1991). We use our current result, $\Lambda^{2}=6.92$ for the square of the integral of the overlap of the proton and deuteron wave functions, which is about $2 \%$ smaller than the previous best estimate $\Lambda^{2}=7.08$. Small effects due to electromagnetic radiative corrections are taken into account by using $f t$ values for nuclear beta decays which have not 
been altered by theoretical corrections for radiative processes (Bahcall and May 1968).

We focus on $\Lambda^{2}$, the square of the overlap integral (at zero energy) where (Salpeter 1952; Bahcall and May 1969):

$$
\Lambda=\left(\frac{a_{p}^{2} \gamma^{3}}{2}\right)^{1 / 2} \int u_{d}(r) u_{p p}(r) d r .
$$

The quantity $a_{p}$ is the $p p$ scattering length, and $\gamma=\left(2 \mu E_{d}\right)^{1 / 2}$ is the deuteron binding wave number (where $\mu$ is the proton-neutron reduced mass and $E_{d}$ is the deuteron binding energy). The function $u_{p p}(r)$ is the radial part of the initial $p p$ wave function and $u_{d}(r)$ is the radial part of the $S$-state deuteron wave function.

In the most systematic previous study, Bahcall and May (1969) carried out calculations for a number of different nuclear potentials and wave functions and found $\Lambda^{2}=7.08(1 \pm 0.025)$. Since 1969, there have been several published calculations of $\Lambda^{2}$ (summarized in Table II of Bahcall and Pinsonneault 1992) with specific assumptions about the nuclear potentials; the values obtained in all but one case ranged from 6.83 to 7.04. Only the calculation by Gould and Guessoum (1990; Guessoum 1988) gives a larger value $\left(\Lambda^{2}=7.39\right)$ than the best estimate of Bahcall and May. (We discuss problems with the Gould-Guessoum calculation at the end of Section 5.) In some of the previous calculations, the effect of vacuum polarization was included or partially included, but was not isolated, which makes comparison between the different calculations difficult.

We first calculate $\Lambda^{2}$-without including vacuum-polarization effects-using improved data for $p p$ scattering and for the deuteron wave function. We investigate systematically the uncertainties in our result, which are caused in part by errors in the experimental measurements of various input parameters and in part by the imprecisely known shapes of the $p p$ and $n p$ nuclear potentials. The theoretical uncertainty is smaller than is suggested by a naive comparison of the different values for $\Lambda^{2}$ obtained using the nuclear potentials available in the literature. Each potential in the literature predicts slightly different values for the measured quantities. If we constrain each nuclear potential to reproduce the same values for the measured parameters, the apparent differences in the calculated values of $\Lambda^{2}$ are 
reduced. We find $\Lambda_{\text {no vac. pol. }}^{2}=6.96$. The uncertainty due to experimental errors is about $0.2 \%$ and that due to theoretical uncertainties is about $1.0 \%$.

We then include the effect of vacuum polarization in a self-consistent way by correcting the nuclear parameters derived from low-energy $p p$ scattering data and by including vacuum polarization in the Schrodinger equation used to calculate the $p p$ wave function. The first effect raises the value of $\Lambda^{2}$ by $0.4_{-0.1}^{+0.4} \%$ and the second correction decreases $\Lambda^{2}$ by about $1.0 \%$. Both effects must be included in a self-consistent treatment. Therefore, vacuum polarization decreases the calculated value of $\Lambda^{2}$ by about $0.6_{-0.4}^{+0.1} \%$. The final result is

$$
\Lambda^{2}=6.92 \times\left(1 \pm 0.002_{-0.009}^{+0.014}\right),
$$

where the first uncertainty is due to experimental errors and the second is due to uncertainties in the nuclear potential.

In Section 2, we discuss the effective-range approximation and use it to estimate the uncertainty in $\Lambda^{2}$ due to the errors in the input parameters that are measured. In Section 3, we discuss the $p p$ wave functions, and in Section 4, we discuss the deuteron wave functions. Our numerical results are presented in Section 5. In Section 6, we discuss the effect of including vacuum polarization in our analysis, and in Section 7, we summarize our results and discuss briefly implications for predicted solar-neutrino rates.

\section{EFFECTIVE-RANGE APPROXIMATION}

Before presenting our numerical results for $\Lambda^{2}$ it is useful to recall the calculation of $\Lambda^{2}$ based on the effective-range approximation (Salpeter 1952; Ellis and Bahcall 1968; Bahcall and May 1969).

If $\rho_{p}$ and $\rho_{d}$ are the $p p$ and deuteron effective ranges, $a_{p}$ is the proton scattering length, and $\gamma$ is the deuteron binding wave number, then

$$
\Lambda_{e f f}(0)=\widetilde{\Lambda}(0)+\left(\frac{\gamma^{2} a_{p} N}{4}\right)\left(\rho_{d}+\rho_{p}\right),
$$

where

$$
\widetilde{\Lambda}(0)=N\left\{1+\frac{a_{p}}{R}\left[E_{1}(\chi)-\frac{e^{-\chi}}{\chi}\right]\right\} e^{\chi},
$$

and $N=\left[\left(1+\eta_{d}^{2}\right)\left(1-\gamma \rho_{d}\right)\right]^{-1 / 2}$. Here, $\eta_{d}$ is the asymptotic ratio of $D$ - to $S$-state 
deuteron wave functions, and $\chi=(\gamma R)^{-1}$.

The low-energy scattering parameters $a_{p}$ and $\rho_{p}$ that are used in Eqs. (4) and (5) are those determined by fitting low-energy $p p$ phase shifts measured relative to a pure Coulomb potential [see Eq. (11) below]. Published values of $a_{p}$ and $\rho_{p}$ that appear in the literature are often corrected for various electromagnetic and/or strong-interaction effects. These corrected values of $a_{p}$ and $\rho_{p}$ cannot be used here. Using $a_{p}=-7.8196(26) \mathrm{fm}, \rho_{p}=2.790(14) \mathrm{fm}$ (Bergervoet et al., 1988), $E_{d}=2.224575(9) \mathrm{MeV}, \eta_{d}=0.0256(4)$, and $\rho_{d}=1.759(5)$ (Brandenburg et al., 1988), we find

$$
\Lambda_{e f f}^{2}(0)=6.975 \times\left[1-0.29\left(\rho_{d}-1.759\right)-0.10\left(\rho_{p}-2.790\right)\right] .
$$

We have exhibited in Eq. (6) the dependence of the result on the effective ranges, which are the experimental quantities with the largest uncertainties. The uncertainty in $\Lambda^{2}$ due to the errors in the experimentally determined quantities is $0.2 \%$ and comes predominantly - but not exclusively - from the imperfect experimental knowledge of $\rho_{d}$ and $\rho_{p}$.

The effective-range approximation yields a value for $\Lambda^{2}$ consistent with what is obtained numerically by integrating the Schrodinger equation $(\sim 6.96$; see below and Bahcall and May 1969). The analytic result given in Eq. (6) does not assume any specific knowledge of the shape of the nuclear potential, reflecting the fact that most of the overlap between the proton and the deuteron wave functions occurs at radii large compared with the range of the nuclear forces. Since the wave functions at asymptotically large radii are accurately determined by the experimentally determined quantities $\gamma, \eta_{d}, \rho_{d}, \rho_{p}$, and $a_{p}$ (Bahcall and May, 1969), $\Lambda^{2}$ is to a large extent determined by the experimental quantities and is therefore insensitive to the details of the nuclear interaction. This robustness will be quantified further in the following sections (see especially the last columns of Table 1 and Table 2).

Since the effective-range approximation gives an answer in agreement with the numerical integrations, we assume that the effect of small changes in the experimental parameters on $\Lambda^{2}$ may be estimated well by the effective-range approximation. This assumption has been verified quantitatively by comparing changes in the calculated $\Lambda^{2}$ produced by varying input parameters in simple wave functions. We conclude that the experimental uncertainty in $\Lambda^{2}$ is indeed about $0.2 \%$. 


\section{PROTON-PROTON WAVE FUNCTION}

We begin by summarizing the theory of low-energy $p p$ scattering without including vacuum polarization. The $s$-wave $p p$ radial wave function $u_{p p}(r)$ satisfies the radial Schrodinger equation,

$$
\frac{d^{2} u}{d r^{2}}-\left[\frac{1}{R r}+V(r)\right] u=-k^{2} u
$$

where $R=\hbar^{2} / M e^{2}=28.8198 \mathrm{fm}, M$ is the proton mass, $V(r)$ is the nuclear potential multiplied by $M / \hbar^{2}$, and $k=M v / 2 \hbar$ is the center-of-mass momentum where $v$ is the relative velocity. The first boundary condition is that $u_{p p}(0)=0$ and the second condition is that $u_{p p}(r)$ approaches a properly normalized distorted Coulomb wave at large $r$.

Since $V(r)$ has finite range, $u_{p p}(r)$ approaches an asymptotic limit, $\phi(r)$, for large radii. In this domain, $\phi(r)$ satisfies

$$
\frac{d^{2} \phi}{d r^{2}}-\left[\frac{1}{R r}\right] \phi=-k^{2} \phi
$$

This equation is solved by

$$
\phi(r)=C_{0}\left[G_{0}(k r)+\cot \delta F_{0}(k r)\right]
$$

where $G_{0}$ and $F_{0}$ are the irregular and regular Coulomb wave functions, and $\delta$ is a phase shift. The Gamow penetration factor is

$$
C_{0}^{2}=\frac{2 \pi \eta}{\exp (2 \pi \eta)-1}
$$

where $\eta=e^{2} / \hbar v$, and $v$ is the relative velocity of the two protons. Note that the normalization of $\phi$ is such that $\phi(0)=1$. For a given potential, $V(r)$, the phase shifts for each energy, $\delta(k)$, are calculated by setting $u_{p p}(0)=0$, numerically integrating Eq. (7) well beyond the range of $V(r)$, and then matching the solution onto Eq. (9). 
The scattering length and effective range are determined for the measured phase shifts in low-energy $p p$ scattering experiments. The scattering length, $a_{p}$, and effective range, $\rho_{p}$, are related to the phase shifts by

$$
C_{0}^{2} k \cot \delta+\frac{1}{R} h(k R)=-\frac{1}{a_{p}}+\frac{1}{2} \rho_{p} k^{2}+\cdots,
$$

where $h(x)=\left(x^{2} / 3\right)\left[1+\left(x^{2} / 10\right)+\ldots\right]$ for small $x$. Since the scattering length is given by the limit

$$
-\frac{1}{a_{p}}=\lim _{k \rightarrow 0} C_{0}^{2} k \cot \delta
$$

only the $k \rightarrow 0$ solutions to Eqs. (7) and (8) are needed to determine $a_{p}$. For $k=0$, Eq. (8) is solved by modified Bessel functions, and one finds (using the definition of $a_{p}$ ) that the $k=0$ limit of Eq. (9) is

$$
\phi(r)=y_{1}(r / R)-\frac{R}{a_{p}} y_{2}(r / R),
$$

where

$$
y_{1}(x) \equiv 2 \sqrt{x} K_{1}(2 \sqrt{x}), \quad \text { and } \quad y_{2}(x) \equiv \sqrt{(x)} I_{1}(2 \sqrt{x}) .
$$

Given $V(r)$, the scattering length is then given by evaluating

$$
\frac{1}{a_{p}}=\frac{2}{R} \frac{r K_{0}(2 \sqrt{r / R})+\alpha \sqrt{r R} K_{1}(2 \sqrt{r / R})}{\alpha \sqrt{r R} I_{1}(2 \sqrt{r / R})-r I_{0}(2 \sqrt{r / R})}
$$

at some $r$ large compared with the range of nuclear forces. Eq. (14) converges to $a_{p}$ as $r$ is increased. In practice, a radius $r \gtrsim 20 \mathrm{fm}$ yields a precision better than that which is determined experimentally. The logarithmic derivative $\alpha=r u_{p p}^{\prime}(r) / u_{p p}(r)$ is determined by solving Eq. (7) numerically with $u_{p p}(0)=0$.

The effective range $\rho_{p}$ is defined by the relation

$$
\rho_{p}=2 \int_{0}^{\infty}\left[\phi^{2}(r)-u^{2}(r)\right] d r
$$

and can be determined numerically for a given potential $V(r)$. 
To obtain the $p p$ wave function, we need to assume a form for the nuclear potential that yields the measured values of $a_{p}$ and $\rho_{p}$, and then integrate the Schrodinger equation. We want our estimate of the uncertainties to be conservative; therefore, we make minimal assumptions about the nuclear potential. Following the example of Bahcall and May (1969), we consider several plausible functional forms for $V(r)$. To fit the two experimental quantities, $a_{p}$ and $\rho_{p}$, each potential is described by two parameters.

The nuclear interaction is heuristically represented by an attractive potential of depth $V_{0}$ and range $b$. We try the following forms for the nuclear potential: (i) a square well potential,

$$
V(r)= \begin{cases}V_{0} & \text { for } r<b \\ 0 & \text { for } r>b\end{cases}
$$

(ii) a Gaussian potential,

$$
V(r)=V_{0} \exp \left[-(r / b)^{2}\right]
$$

(iii) an exponential potential,

$$
V(r)=V_{0} \exp (-r / b)
$$

(iv) a Yukawa potential,

$$
V(r)=V_{0}(b / r) \exp (-b / r)
$$

and (v) a repulsive-core ( $\mathrm{RC})$ potential,

$$
V(r)=V_{0} \exp (-r / b)+300 \mathrm{MeV} \Theta(0.4 \mathrm{fm}-r),
$$

where $\Theta$ is a step function. Three of these shapes (square well, Yukawa, and exponential) were used by Bahcall and May (1969). The values of $V_{0}$ and $b$ that give $a_{p}=-7.8196 \mathrm{fm}$ and $\rho_{p}=2.790$ (see Section II) fm are listed in Table I for the five models, and the potentials are plotted in Fig. 1. 


\section{THE DEUTERON WAVE FUNCTION}

The deuteron wave function is determined by a procedure similar to that used to calculate the $p p$ wave functions. To estimate the sensitivity of $\Lambda^{2}$ to details of the neutron-proton interaction, we use six wave functions which fit the static deuteron parameters (e.g., magnetic and electric quadrupole moments), as well as those which are more crucial to the calculation of $\Lambda^{2}: E_{d}, \eta_{d}$, and $\rho_{d}$, [see Eqs. (4) and (5), and the following discussion]. We are grateful to R. Wiringa for supplying a code that calculates the deuteron wave functions. The wave functions are obtained from the Argonne v14 potential (Wiringa et al., 1984), the Reid soft-core (RSC) potential (Reid, 1968), the Urbana v14 potential (Lagaris and Pandharipande, 1981), the super-soft-core (SSC) potential (de Tourreil and Sprung, 1973), and the Argonne v18 potential (Wiringa, 1993); in addition, we also calculate $\Lambda^{2}$ with the McGee wave function (McGee, 1966), which was used by Bahcall and May.

The six wave functions are plotted in Fig. 2, and the values of $E_{d}, \eta_{d}$ and $\rho_{d}$ are given in Table 2. Since the potentials used to calculate the wave functions were constructed by assuming different values for the deuteron properties, none of the potentials precisely reproduces the current values of all of the experimental quantities of relevance here. Therefore, the uncertainties in the wave functions due to uncertainties in the details of the nuclear interaction are not as large as suggested by the (already small) differences in the wave functions plotted in Fig. 2. For example, the asymptotic behavior of the wave function is given by $u_{d}(r) \rightarrow N(2 \gamma)^{1 / 2} \exp (-\gamma r)$, where $N$ and $\gamma$ are fixed by $E_{d}, \rho_{d}$ and $\eta_{d}$. Since these primary quantities are determined accurately by the existing experimental data [see discussion following Eqs. (4) and (5)], the behavior of the wave function at large radii is not as uncertain as suggested by Fig. 2. Most of the overlap integral occurs at large radii. The value of $\Lambda^{2}$ that is obtained by numerically integrating the overlap of $u_{d}$ and $u_{p p}$, when $u_{d}$ has an incorrect asymptotic behavior, is inconsistent with current knowledge. In order to obtain an accurate value of $\Lambda^{2}$ that uses the current experimental data, we make small corrections with the aid of the effective-range approximation. We noted earlier (end of Section II) that the effective-range approximation reliably reflects small changes in $\Lambda^{2}$ due to small changes in the parameters. Specifically, we evaluate $\Lambda^{2}$ for a given deuteron 
potential from the relation,

$$
\Lambda^{2}=\left[\frac{\Lambda_{\text {eff }}^{2}(\text { experimental parameters })}{\Lambda_{e f f}^{2}(\text { model parameters })}\right] \Lambda_{\text {num }}^{2} \equiv C \Lambda_{\text {num }}^{2}
$$

where $\Lambda_{\text {num }}^{2}$ is the value obtained numerically by calculating the overlap integrals with the six deuteron wave functions, and the values of $\Lambda_{e f f}$ are obtained from the effective-range equations, Eqs. (4) and (5). For the potentials listed in Table 2, the value of $C$ ranges from 0.979 to 0.996 . By using several different potentials, we probe the sensitivity of $\Lambda^{2}$ to details of the nuclear interaction.

\section{NUMERICAL RESULTS FOR $\Lambda^{2}$}

In Fig. 3, we plot the integrand $u_{p p}(r) u_{d}(r)$ as a function of radius for the five assumed $p p$ potentials (using the Argonne v14 deuteron potential). This figure shows that drastic changes in the shape of the $p p$ interaction [see Fig 1] result in relatively small changes in the value of the integrand, and the difference is significant only in the region $r \lesssim 5 \mathrm{fm}$. The integrand is insensitive to the shape of the $p p$ potential for radii $r \gtrsim 5 \mathrm{fm}$. In the region where there is a visible difference $(r \lesssim 5 \mathrm{fm})$, the wave functions are constrained by the measured effective range [see Eq. (15)], so those wave functions which are smaller in the region $r \lesssim 2 \mathrm{fm}$ are larger in the region $r \gtrsim 2 \mathrm{fm}$ (and vice versa). About $40 \%$ of the integrand comes from the region $r \lesssim 5 \mathrm{fm}$ and only about $2 \%$ comes from the region $r \lesssim 1 \mathrm{fm}$.

The values of $\Lambda^{2}$ obtained using the five different $p p$ interactions are listed in Table I. (Here we used the Argonne v14 deuteron potential.) They range from $\Lambda^{2}=6.916$ (using the square-well potential) to $\Lambda^{2}=6.979$ (using the Yukawa potential) to $\Lambda^{2}=6.939$ (using the strong repulsive core). The difference in $\Lambda^{2}$ between the pure exponential potential and the potential that includes a strong repulsive core is only $0.3 \%$. Thus we assign a total uncertainty of $\pm 0.5 \%$ to the value of $\Lambda^{2}$ due to uncertainty in the details of the $p p$ interaction. The exponential potential yields a central value of $\Lambda^{2}=6.960$.

Next, we determine the spread in the values of $\Lambda^{2}$ caused by different deuteron potentials (using the exponential $p p$ potential). The results are listed in Table II and range from $\Lambda^{2}=6.917$ (for the RSC potential) to $\Lambda^{2}=6.988$ (for the Argonne 
v18 potential). We also assign an uncertainty of $\pm 0.5 \%$ to the value of $\Lambda^{2}$ due to uncertainty in the deuteron wave function. The Argonne v14 potential yields the central value of $\Lambda^{2}=6.960$.

In the column labeled $\Lambda_{\text {num }}^{2}$ in Table 2, we list the values of $\Lambda^{2}$ obtained by naively inserting the various tabulated deuteron wave functions into the overlap integral. These values vary from 6.915 to 7.129 , which would naively imply an uncertainty of about $\pm 1.5 \%$. However, when the values of $\Lambda^{2}$ are corrected for the differences in the assumed values of $E_{d}, \rho_{d}$, and $\eta_{d}$, the resulting total uncertainty is only $\pm 0.5 \%$. The magnitude of the uncertainty in $\Lambda^{2}$ due to uncertainty in the shape of the deuteron potential is similar to that due to uncertainty in the shape of the $p p$ potential.

The differences in the various published values for $\Lambda^{2}$ (see Table II in Bahcall and Pinsonneault 1992) are primarily the result of differences in the input parameters. The theoretical uncertainty is smaller than one would estimate by simply comparing the published results. For example, the result given here is almost $2 \%$ smaller than that obtained by Bahcall and May (1969). This difference can be traced primarily to the high value of $E_{d}$ embodied in the deuteron wave function used therein (from McGee 1966, who adopted $E_{d}=2.267 \mathrm{MeV}$ ), and secondarily to smaller differences in the input $\eta_{d}, \rho_{d}, \rho_{p}$, and $a_{p}$. If we correct for these discrepancies in input data using the effective-range approximation, the Bahcall-May result becomes 7.02 , within $1 \%$ of the current result.

The anomalously high value of $\Lambda^{2}=7.39$ obtained by Gould and Guessoum (1990; Guessoum 1988) deserves special comment. The authors state that this result was obtained using the overlap of the Paris deuteron wave function (Lacombe et al. 1980) and a $p p$ wave function employing a Bargmann nuclear potential (see Noyes 1967) modified to include a soft repulsive core. From the discussion in the papers by Gould and Guessoum, it not clear what form they assumed for the $p p$ potential, nor is it clear what values of $a_{p}$ and $\rho_{p}$ they adopted. Therefore, it is difficult to make an unambiguous comparison with their result or even to understand precisely the origin of the difference between the Gould-Guessoum value and all the other values given in Table 1 and Table 2 (as well as the other values obtained by different authors; see Table II of Bahcall and Pinsonneault 1992). We have, however, carried out an illustrative calculation using a potential 
that seems to be suggested by the description in Gould and Guessoum. We obtain a value of 7.42 for $\Lambda^{2}$, consistent with the Gould-Guessoum value, by adding to our exponential potential an attractive square-well core of depth $30 \mathrm{MeV}$ and range $0.4 \mathrm{fm}$. However, for this potential, we find values of $a_{p}=-8.4826 \mathrm{fm}$ and $\rho_{p}=2.727 \mathrm{fm}$, which are inconsistent with the experimental data (discrepancies $>200 \sigma$ and $4 \sigma$ for $a_{p}$ and $\rho_{p}$, respectively). We do not claim that this procedure was used by Gould and Guessoum, but it is the only procedure we could think of that reproduces their result. In all of the numerical experiments that we have performed using wave functions and potentials that are consistent with the current experimental data, we have never obtained a value for $\Lambda^{2}$ greater than 7.00. We conclude that the Gould-Guessoum value can only be obtained by using some input data or some assumption that contradicts the existing experimental information on the $p p$ system.

\section{VACUUM POLARIZATION}

We next consider, following Bohannon and Heller (1977) and Gould (1991), the effect of vacuum polarization (VP) on the $p p$ wave function. In quantum electrodynamics, the Coulomb potential is obtained from the Fourier transform of the matrix element for scattering via exchange of one virtual photon from an electrostatic source. To next order in $\alpha$, the photon propagator is augmented by an electron-positron loop. This augmentation introduces an $\mathcal{O}(\alpha)$ correction to the matrix element which, when Fourier transformed, leads to a small correction, the Uehling potential (Uehling, 1935), to the electrostatic potential. The complete electrostatic potential becomes, in this approximation,

$$
\frac{e^{2}}{r}+\frac{e^{2}}{r}\left(\frac{2 \alpha I(r)}{3 \pi}\right)
$$

where

$$
I(r)=\int_{1}^{\infty} e^{-2 m_{e} r x}\left(1+\frac{1}{2 x^{2}}\right) \frac{\left(x^{2}-1\right)^{1 / 2}}{x^{2}} d x .
$$

The function $I(r)$ has the limiting forms

$$
I(r)=-\gamma-5 / 6-\ln \left(m_{e} r\right), \quad \text { for } \quad m_{e} r \ll 1 \text {, }
$$


and

$$
I(r)=\frac{3(2 \pi)^{1 / 2}}{4} \frac{e^{-2 m_{e} r}}{\left(2 m_{e} r\right)^{3 / 2}}, \quad \text { for } \quad m_{e} r \gg 1
$$

The function $I(r)$ has a logarithmic singularity for very small radii, is of order a few until a radius of about $1 / 2 m_{e}=193.1 \mathrm{fm}$, and then suffers an exponential falloff (arising from the exchange of a virtual electron-positron pair) for $r \gtrsim 1 / 2 m_{e}$.

A self-consistent determination of the effect of VP on the calculation of $\Lambda^{2}$ must take into account the following two effects. First, VP must be incorporated in the analysis of low-energy $p p$ scattering data, which alters the inferred parameters in the nuclear potential. Second, the $p p$ wave function must be calculated with the Uehling potential in the Schrodinger equation. Using a sophisticated effectiverange formalism for vacuum polarization, Bohannon and Heller (1977) find that including VP in the potential in the Schrodinger equation decreases the $p p$ reaction rate by between $0.8 \%$ and $1.2 \%$, and using the WKB approximation, Gould (1990) finds that VP decreases the $p p$ reaction rate by about $1.3 \%$. However, in both of these papers, the quoted result includes only the second effect described above, i.e., the inclusion of the Uehling potential in the calculation of the $p p$ wave function. Neither paper includes a calculation of the effect of VP on the low-energy scattering parameters from which the nuclear potential is determined.

We perform a numerical calculation of the effect of $\mathrm{VP}$ on the $p p$ reaction rate which allows us to isolate both effects of VP. First, we consider the effect of VP on the low-energy $p p$ scattering parameters, $a_{p}$ and $\rho_{p}$, that are obtained experimentally. These parameters are fit to phase shifts measured at energies of roughly $0.3 \mathrm{MeV}$ to $30 \mathrm{MeV}$. The effective-range approximation converges only for inverse wavenumbers $k^{-1}$ small compared with the range of the nuclear potential. Since the range of the Uehling potential is $\sim 200 \mathrm{fm}$, we cannot estimate the effect of the Uehling potential on $a_{p}$ and $\rho_{p}$ by using Eqs. (14) and (15), and incorporating the Uehling potential together with the nuclear potential. We must calculate the effect of VP on the phase shifts for the energies at which the measurements are performed.

For a given nuclear potential, the phase shifts are determined by integrating 
the Schrodinger equation, Eq. (7). To account for VP, we make the substitution

$$
V(r) \rightarrow V(r)\left[1+\frac{2 \alpha I(r)}{3 \pi}\right]
$$

in Eq. (7). We call the phase shifts obtained from this substitution $\delta_{V P}(k)$, and we call the phase shifts obtained from Eq. (7) (without VP) $\delta(k)$. In fact, the measured phase shifts are $\delta_{V P}$, and the low-energy scattering parameters are correctly obtained by fitting to the relation

$$
C_{0}^{2} k \cot \delta_{V P}(k)+\frac{1}{R} h(k R)=-\frac{1}{a_{p}}+\frac{1}{2} \rho_{p} k^{2},
$$

instead of to Eq. (11). The $p p$ potentials used in Section III were constructed using scattering parameters obtained from Eq. (11), not Eq. (27). Therefore, in constructing the $p p$ nuclear potential as in Section III, we make the substitutions $a_{p} \rightarrow a_{p}-\delta a_{p}$ and $\rho_{p} \rightarrow \rho_{p}-\delta \rho_{p}$ in the low-energy parameters, where $\delta a_{p}$ and $\delta \rho_{p}$ are obtained by fitting the measured phase shifts to

$$
C_{0}^{2} \cot \delta_{V P}+\frac{1}{R} h(k R)-f(k)=-\frac{1}{a_{p}-\delta a_{p}}+\frac{1}{2}\left(\rho_{p}-\delta \rho_{p}\right) k^{2} .
$$

Here, the function

$$
f(k) \equiv C_{0}^{2} k\left[\cot \delta_{V P}(k)-\cot \delta(k)\right]
$$

is obtained for each data point by integrating the Schrodinger equation numerically with and without the Uehling potential using a nuclear potential that gives the correct $a_{p}$ and $\rho_{p}$. We used an exponential nuclear potential, but the results are insensitive (to an accuracy of about $0.1 \%$ in $a_{p}$ and $\rho_{p}$ ) to the choice of potential.

We first calculate $\delta a_{p}$ and $\delta \rho_{p}$ with the aid of a set of measured phase shifts given by Jackson and Blatt (1950). Although the modern data set consists of more data points, the Jackson-Blatt data are sufficient (see discussion below of results using part of the data and using simulated data) to evaluate the small effect of VP on the scattering parameters. By first fitting the complete data set to Eq. (27), and then to Eq. (29), we find $\delta a_{p}=0.0687$ and $\delta \rho_{p}=-0.030$. Using Eq. (4), we find that this results in an increase in $\Lambda^{2}$ of $0.7 \%$. The sign of this result is expected: If VP (a repulsive potential) is included, the nuclear potential must be 
deeper to compensate, so $\Lambda^{2}$ is increased. To assess the dependence of our results on the data set used, we do the same with only the lower-energy data points. (The complete data set consists of 24 data points at center-of-mass energies of 0.1765-3.53 MeV obtained with Van de Graaff generators, and five data points with energies of 4.2-14.5 MeV obtained with cyclotrons. In their analysis, Jackson and Blatt disregarded the data points obtained with cyclotrons since the data were less reliable at the time.) Using only the lower-energy data, we find $\delta a_{p}=0.0732$ and $\delta \rho_{p}=-0.042$, which leads to an $0.4 \%$ increase in $\Lambda^{2}$.

We used simulated data to estimate the effect of VP on the modern data set, which include measurements up to $30 \mathrm{MeV}$. We calculated the effect of VP on the values of $a_{p}$ and $\rho_{p}$ obtained from ten simulated data points that exactly reproduce the measured $a_{p}$ and $\rho_{p}$ in Eq. (11) which are uniformly spaced between 3 and 30 $\mathrm{MeV}$. This calculation gives the correct fractional changes due to VP if the changes are small. For this simulated modern data set, $\delta a_{p}=0.0321$ and $\delta \rho_{p}=-0.001$, which again results in an increase in $\Lambda^{2}$ of $0.4 \%$. Since this data set most-closely resembles the modern data set, we choose $0.4 \%$ to be the best estimate of the correction to $\Lambda^{2}$ due to VP-corrections to the low-energy scattering parameters. To be conservative, we estimate that the total theoretical uncertainty includes the full range inferred above using the Jackson-Blatt data and also includes a small uncertainty due to the choice of the nuclear potential. We conclude that correcting the low-energy scattering parameters for VP results in an $0.4_{-0.1}^{+0.4} \%$ increase in $\Lambda^{2}$.

Next we evaluate the effect of including VP on the wave function obtained by integrating the Schrodinger equation. By numerically integrating Eq. (7) with and without the Uehling potential, but using the same nuclear potential, we find that the value of $\Lambda^{2}$ is decreased by $0.9 \%$ when the Uehling potential is included. The sensitivity of this result to the choice of the shape of the nuclear potential is less than $0.1 \%$. Again, the sign of the effect is expected since the Uehling potential is repulsive. This is the result for the correction to $\Lambda^{2}(0)$, the matrix element squared at zero energy. The most probable energy of interaction in the Sun is about $6 \mathrm{keV}$ (Bahcall 1989). We use the WKB approximation to evaluate the energy dependence of the VP correction to $\Lambda^{2}$ (Gould 1990; Kamionkowski and Bahcall 1993). The magnitude of this VP correction is about $10 \%$ larger at 6 $\mathrm{keV}$ than at zero-energy, so we conclude that inclusion of the Uehling potential in 
the calculation of the wave function decreases $\Lambda^{2}$ by $1.0 \%$ at solar energies. Our result is consistent with that of Bohannon and Heller, but is slightly smaller than Gould's result. This small difference is most likely due to Gould's use of the WKB approximation (see Kamionkowski and Bahcall 1993).

Combining our results from including vacuum polarization in analyzing the scattering data and in integrating Schrodinger's equation, we find that VP decreases $\Lambda^{2}$ by $0.6_{-0.4}^{+0.1} \%$. The net effect we find for VP on the $p p$ matrix element is about half that found by Bohannon and Heller (1977) and Gould (1991). We attribute the difference to the fact that we self-consistently include the effect of VP on the measured low-energy scattering parameters, whereas this aspect of the influence of VP (which partially cancels the effect of VP in the potential used in integrating the Schrodinger equation) was not considered by Gould and was not isolated by Bohannon and Heller.

\section{SUMMARY AND DISCUSSION}

We have calculated the matrix element for the reaction $p+p \rightarrow{ }^{2} \mathrm{H}+e^{+}+\nu_{e}$ with and without the effects of vacuum polarization. Without vacuum polarization, we find the square of the overlap integral to be $\Lambda^{2}=6.96 \times(1 \pm 0.002 \pm 0.010 \%)$, where the first uncertainty results from $1 \sigma$ errors in the experimental quantities and the second reflects imprecise knowledge of the shape of the nuclear potential. We include vacuum polarization self-consistently in the low-energy $p p$ scattering parameters and in the numerical calculation of the $p p$ wave function. We find that vacuum polarization decreases $\Lambda^{2}$ by $0.6_{-0.4}^{+0.1} \%$. Our final result is $\Lambda^{2}=$ $6.92 \times\left(1 \pm 0.002_{-0.009}^{+0.014}\right)$. We show elsewhere that vacuum polarization will decrease the rates for all the other reactions in the $p p$ chain and the CNO cycle by small amounts, less than 5\% (Kamionkowski and Bahcall 1993).

In addition to the $\mathcal{O}(\alpha) \mathrm{VP}$ corrections to the $p p$ potential, there are $\mathcal{O}(\alpha)$ radiative corrections (involving an extra soft photon in the final state) to the $p p$ reaction. One can use the similarity of the radiative corrections to the axial-vector part of neutron decay to those for proton decay in the $p p$ reaction to account for these radiative corrections to the $p p$ reaction (Bahcall and May 1968). This correction has been included in Eq. (1). 
It is conventional to use the low-energy cross-section factor, $S(0)$, in stellarevolution calculations. Following the discussion in Bahcall et al. (1982) and Bahcall and Pinsonneault (1992), we find [cf. Eq. (1)]:

$$
S_{p p}(0)=3.89 \times 10^{-25}(1 \pm 0.011) \mathrm{MeV}-\text { barns } .
$$

In calculating the error given in Eq. (30), we have used $1 \sigma$ errors for experimentallymeasured quantities (such as $G_{A} / G_{V}$ or $a_{p}$ ) and one-third the total range of values for theoretically-calculated effects (such as the uncertainty from nuclear potentials or from mesonic corrections). Although the theoretical errors cannot be used in a precise statistical sense, the uncertainty quoted in Eq. (30) is intended to be used as an approximate $1 \sigma$ error in Monte Carlo studies ( $c f$. Bahcall and Ulrich 1988) of the overall uncertainty in predictions of solar-neutrino event rates. Our best estimate is about 3\% smaller than that quoted by Bahcall and Pinsonneault. This is due in part (2\%) to our improved value for $\Lambda^{2}$, and in part (1\%) to an updated value for $G_{A} / G_{V}$ (Hikasa et al. 1992).

Our results imply a $7.5 \%$ increase in the predicted event rates for the Kamiokande (Hirata et al. 1991) solar-neutrino experiment and a $6 \%$ (0.5 SNU) increase for the chlorine solar-neutrino experiment relative to the calculations of Bahcall and Pinsonneault (1992), slightly increasing the discrepancy between standard-model predictions and observations. The predicted event rate for the Borexino experiment (sensitive to ${ }^{7} \mathrm{Be}$, Raghavan 1990) is increased by about $3 \%$.

It is a pleasure to thank F. Dyson, S. Freedman, R. Gould, W. Haxton, L. Heller, F. Wilczek, and R. Wiringa for valuable conversations and suggestions. We are especially grateful to R. Gould for detailed, constructive comments on a draft version of the manuscript. MK was supported by the Texas National Laboratory Research Commission, and by the DOE through Grant No. DE-FG0290ER40542. JNB was supported by the NSF through Grant No. PHY92-45317. 


\section{REFERENCES}

Bahcall, J. N. 1989, Neutrino Astrophysics (Cambridge University Press, Cambridge).

Bahcall, J. N., and May, R. M. 1968, Ap. J. (Letters), 152, L17.

Bahcall J. N., and May, R. M. 1969, Ap. J. 155, 501.

Bahcall J. N. et al. 1982, Rev. Mod. Phys. 54, 767.

Bahcall J. N., and Ulrich, R. K. 1988, Rev. Mod. Phys. 60, 297.

Bahcall, J. N., and Pinsonneault, M. H. 1992, Rev. Mod. Phys. 64, 885.

Bargholtz, C. 1979, Ap. J. (Letters) 233, L161.

Bergervoet J. R. et al. 1988, Phys. Rev. C 38, 15.

Bethe, H., and Critchfield, C. L. 1938, Phys. Rev. 54, 248.

Blin-Stoyle, R. J., and Papageorgiou, S. 1965, Nucl. Phys. 64, 1.

Bohannon, G. E., and Heller, L. 1977, Phys. Rev. C 15, 1221.

Brandenburg, R. A. et al. 1988, Phys. Rev. C 37, 1245.

Brolley, J. E. 1971, Sol. Phys. 20, 249.

Carlson, J., Riska, D. O., Schiavilla, R., and Wiringa, R. B. 1991, Phys. Rev. C 44,619 .

Dautry, F., Rho, M., and Riska, D. O. 1976, Nucl. Phys. A264, 507.

Davis, R., Jr. 1989, in Proceedings of the Thirteenth International Conference on Neutrino Physics and Astrophysics, Boston, Massachusetts, 5-11 June 1988, edited by J. Schneps et al. (World Scientific, Singapore), p. 518. 
de Tourreil, R., and Sprung, D. W. L. 1973 Nucl. Phys. A201, 193.

Ellis, S. D., and Bahcall, J. N. 1968, Nucl. Phys. A114, 636.

Gari, M. 1978, in Proceedings of Informal Conference on Status and Future of Solar Neutrino Research, edited by G. Friedlander (Brookhaven National Laboratory), Report No. 50879, Vol. 1, p. 137.

Gari, M., and Huffman, A. H. 1972, Ap. J. 178, 543.

Gould, R. J. 1990 Ap. J. 363, 574.

Gould, R. J., and Guessoum, N. 1990, Ap. J. (Letters) 359, L67.

Guessoum, N. 1988, Ph.D Thesis, University of California, San Diego.

Hirata, K. S. et al. 1991, Phys. Rev. D 44, 2241.

Hikasa, K. et al. 1992, Phys. Rev. D 45, S1. 1.

Jackson J. D., and Blatt, J. M. 1950 Rev. Mod. Phys. 22, 77.

Kamionkowski, M., and Bahcall, J. N. 1993, IASSNS-AST-93/21.

Lacombe, M. et al. 1980, Phys. Rev. C 21, 861.

Lagaris, I. E., and Pandharipande, V. R. 1981, Nucl. Phys. A359, 331.

Noyes, H. P. 1968, in Few-Body Problems, Light Nuclei, and Nuclear Interactions, Vol. I, ed. G. Paic and J. Slaus (New York, Gordon and Breach), p. 9.

Raghavan, R. S. 1990, in Proceedings of the 25th International Conference on High Energy Physics, edited by K. Phu and Y. Yamaguchi (World Scientific, Singapore), Vol. I, p. 482.

Reid, R. V. 1968, Ann. Phys. 50, 411. 
Salpeter, E. E. 1952, Phys. Rev. 88, 547.

Uehling, E. A. 1935, Phys. Rev. 48, 55.

Wiringa, R. B., Smith, R. A., and Ainsworth, T. L. 1984, Phys. Rev. C 29, 1207.

Wiringa, R. B. 1993, work in progress. 


\section{FIGURE CAPTIONS}

1) Proton-Proton potentials. The solid curve is the exponential potential, the dot-short-dash curve is the Yukawa potential, the short-dash curve is the Gaussian potential, the long-dash curve is the square-well potential, and the dot-long-dash curve is the repulsive-core potential. All five potentials result in a scattering length $a_{p}=-7.8196$ and an effective range $\rho_{p}=2.790$.

2) Deuteron wave functions. The solid curve is the wave function for the SSC potential, the short-dash-long-dash curve is that for the Urbana v14 potential, the short-dash curve is that for the Argonne v18 potential, the long-dash curve is that for the Argonne v14 potential, the dot-short-dash curve is that for the RSC potential, and the dot-long-dash curve is the McGee wave function.

3) Overlap of $p p$ and deuteron wave functions. As in Fig. 1, the solid curve comes from using the exponential $p p$ potential, the dot-short-dash curve from the Yukawa potential, the short-dash curve from the Gaussian potential, the long-dash curve from the square-well potential, and the dot-long-dash curve from the repulsive-core potential. In all five cases, the Argonne v14 deuteron wave function is used. In (a) we show the overlap out to a radius of $50 \mathrm{fm}$, while in (b) we magnify the first $5 \mathrm{fm}$. 


\begin{tabular}{|c|c|c|c|}
\hline \multicolumn{4}{|c|}{ Proton-Proton Potentials } \\
\hline Potential & $V_{0}(\mathrm{MeV})$ & $b(\mathrm{fm})$ & $\Lambda^{2}$ \\
\hline SW & -11.751 & 2.7718 & 6.916 \\
\hline Gaussian & -27.729 & 1.8912 & 6.937 \\
\hline Exponential & -98.861 & 0.7407 & 6.960 \\
\hline Yukawa & -46.124 & 1.1809 & 6.979 \\
\hline RC & -314.704 & 0.5565 & 6.939 \\
\hline
\end{tabular}

Table 1. Proton-Proton potential parameters. The values of $\Lambda^{2}$ were obtained using the Argonne v14 deuteron potential.

\begin{tabular}{|c|c|c|c|c|c|c|}
\hline \multicolumn{7}{|c|}{ Deuteron Potentials } \\
\hline Potential & $E_{d}(\mathrm{MeV})$ & $\eta_{d}$ & $\rho_{d}(\mathrm{fm})$ & $\Lambda_{\text {num }}^{2}$ & $\Lambda_{\text {eff }}^{2}$ & $\Lambda^{2}$ \\
\hline Experimental & 2.224575 & 0.0256 & 1.759 & & 6.975 & \\
\hline SSC & 2.224066 & 0.0255 & 1.833 & 7.129 & 7.128 & 6.977 \\
\hline Urbana v14 & 2.224637 & 0.0254 & 1.816 & 7.099 & 7.092 & 6.981 \\
\hline Argonne v18 & 2.224575 & 0.0253 & 1.770 & 7.009 & 7.000 & 6.988 \\
\hline Argonne v14 & 2.224884 & 0.0266 & 1.805 & 7.055 & 7.070 & 6.960 \\
\hline RSC & 2.224688 & 0.0262 & 1.758 & 6.915 & 6.973 & 6.917 \\
\hline McGee & 2.2669104 & 0.0269 & 1.749 & 7.026 & 7.031 & 6.970 \\
\hline
\end{tabular}

Table 2. Deuteron potentials. The values of $\Lambda^{2}$ were obtained using the exponential proton-proton potential. 


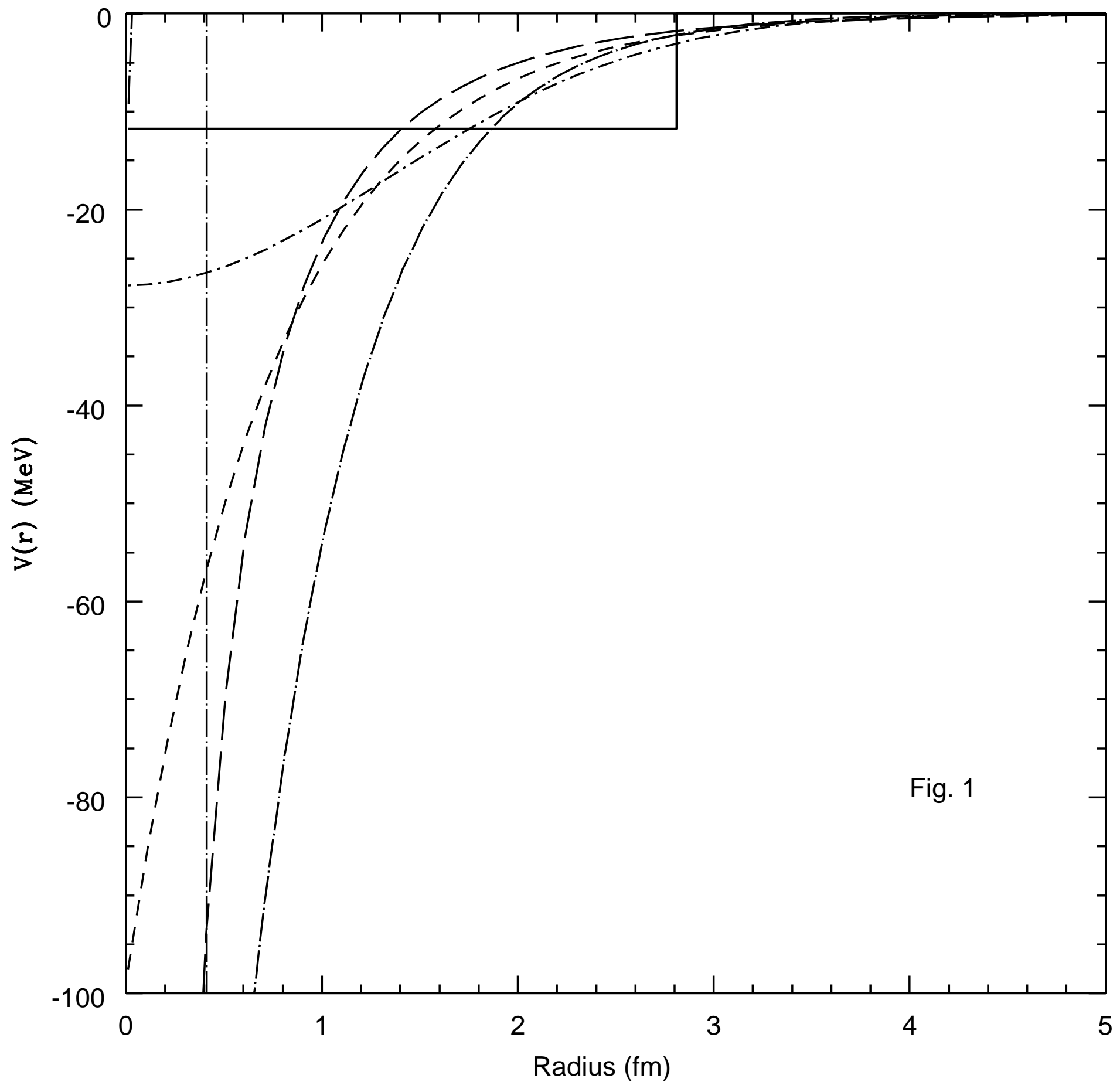




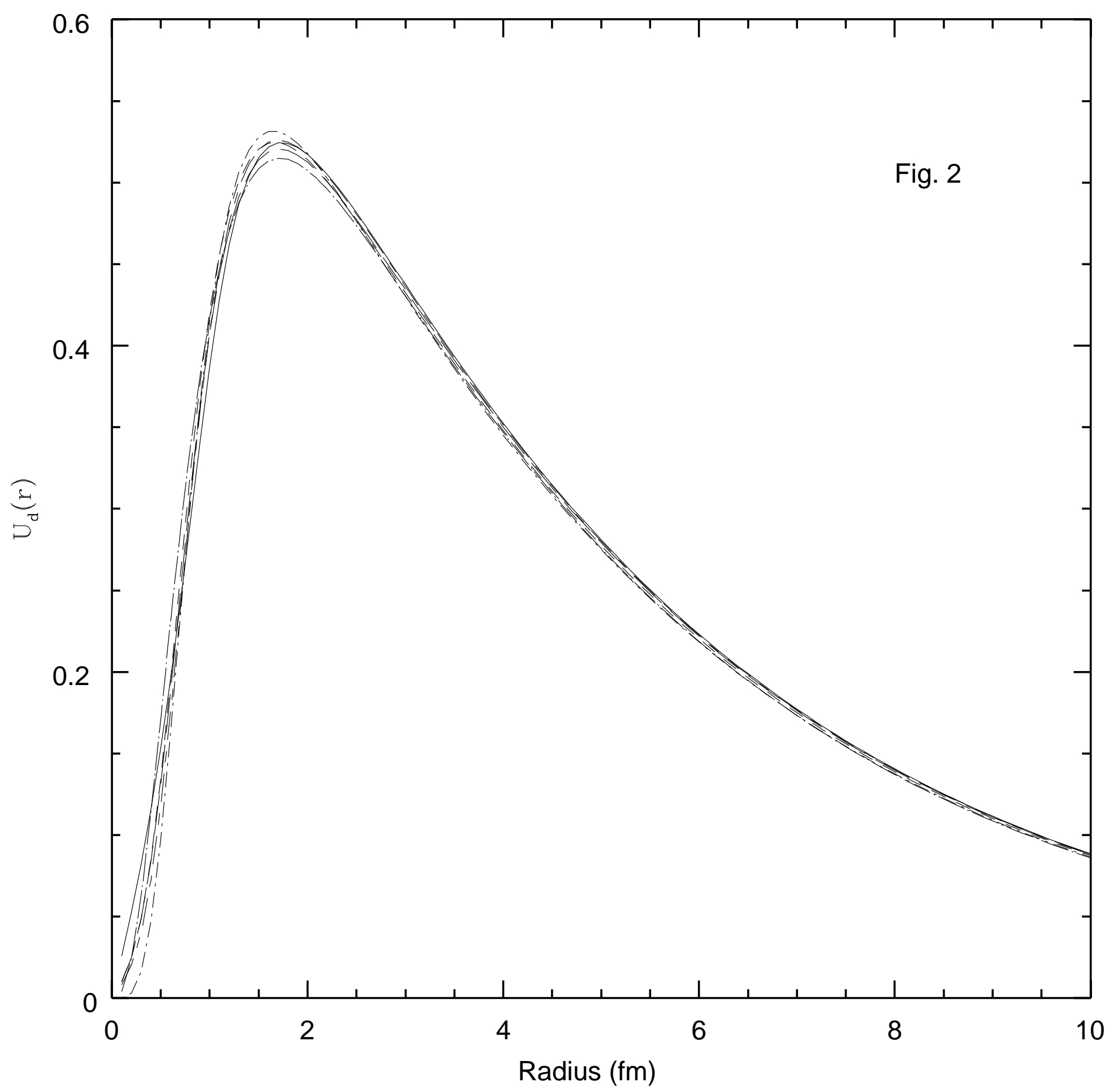




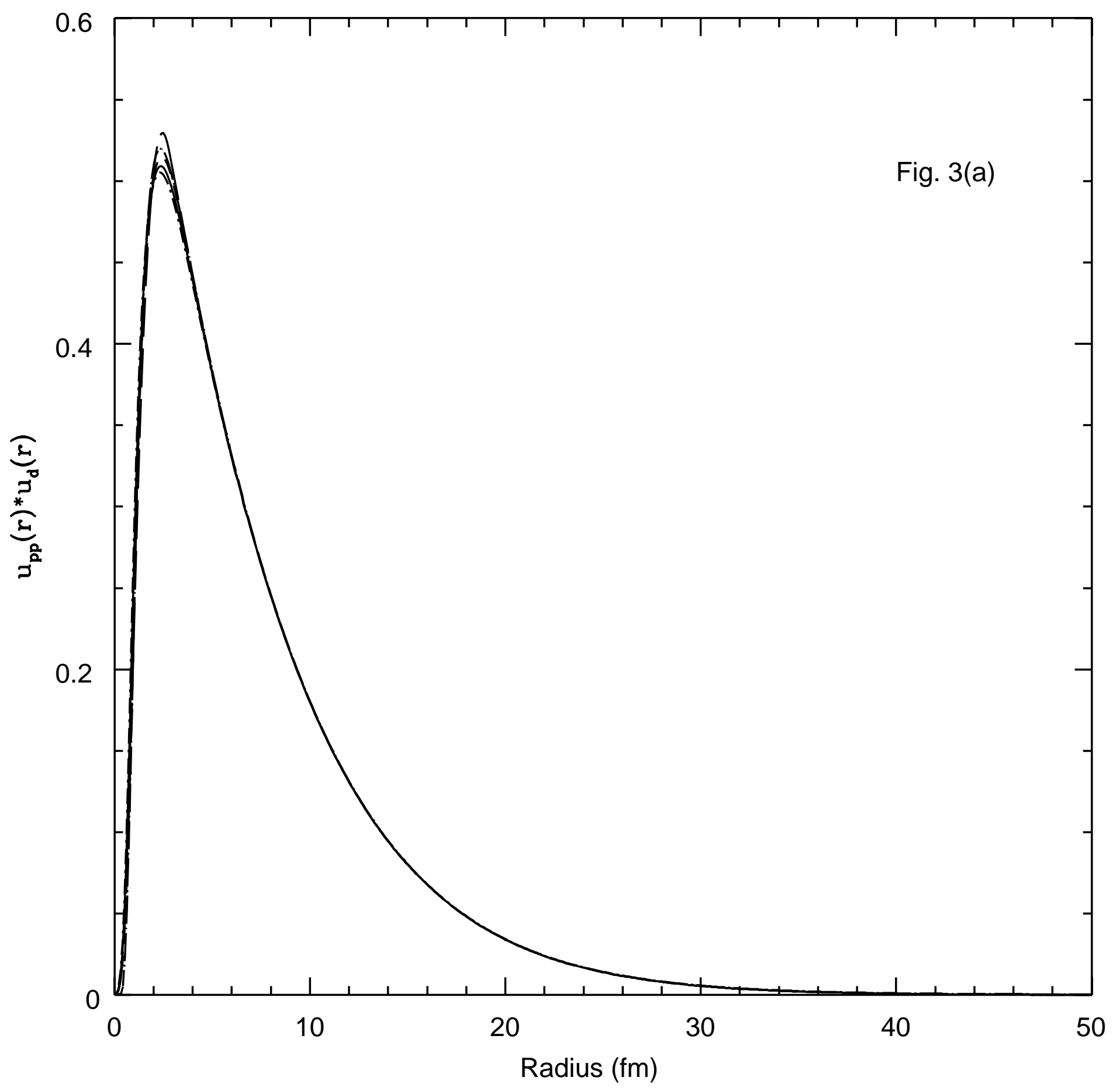




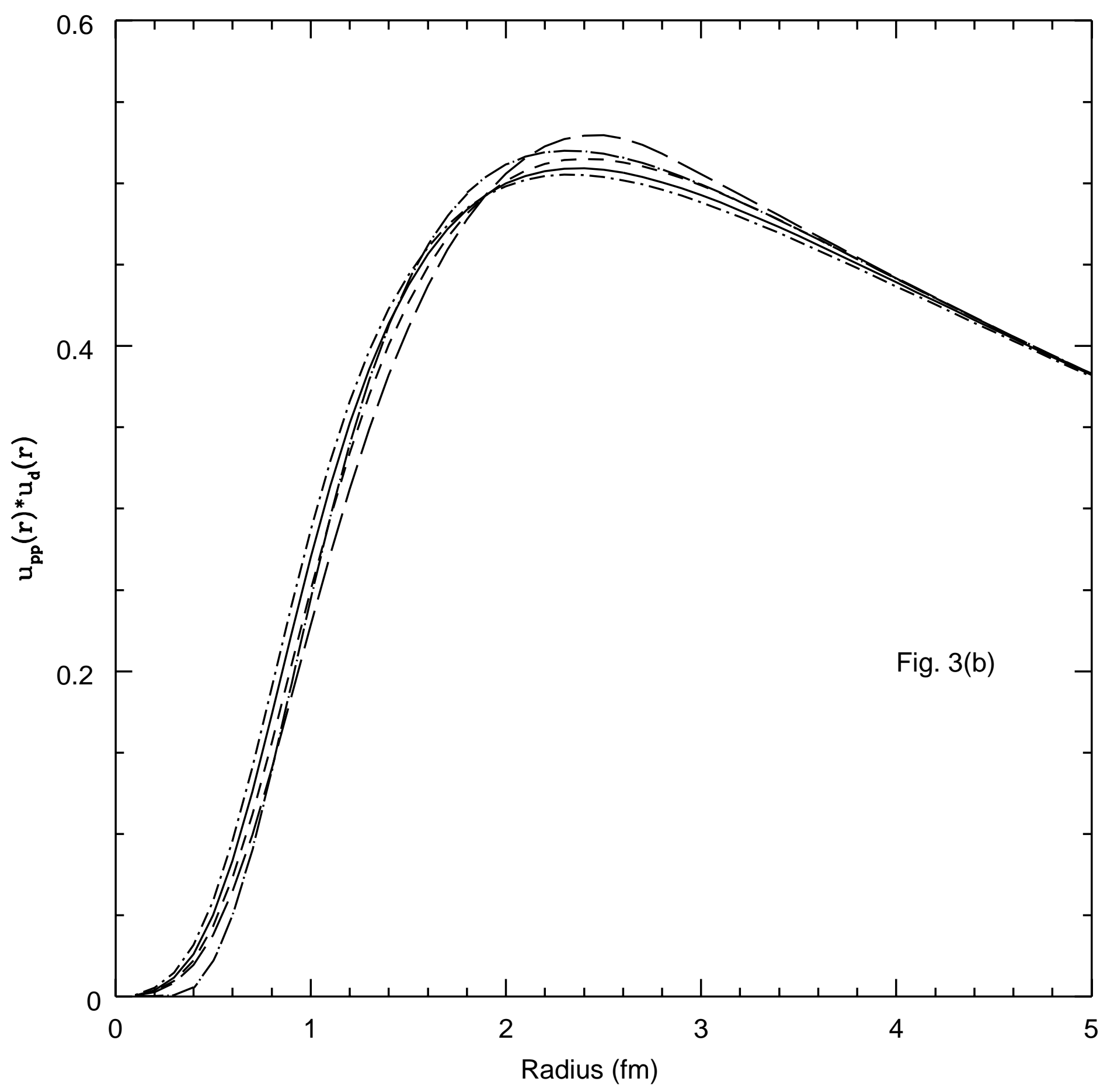

